\title{
Effects of Geon Deletion, Scrambling, and Movement on Picture Recognition in Pigeons
}

\author{
Kim Kirkpatrick-Steger \\ and Edward A. Wasserman \\ University of Iowa
}

\author{
Irving Biederman \\ University of Southern California
}

\begin{abstract}
E. A. Wasserman, K. Kirkpatrick-Steger, L. J. Van Hamme, and I. Biederman (1993) demonstrated that scrambling an object's parts or "geons" (I. Biederman, 1987) produced marked reductions in the pigeon's picture recognition accuracy, indicating that discriminative responding to pictures is controlled by more than simple particulate features. The present effort was designed to further assess the contribution of various stimulus attributes to picture perception. Four pigeons were trained to discriminate 4 line drawings of human-made objects. Subsequent tests revealed that (a) the spatial organization of the geons was a major contributor to picture recognition; (b) the individual geons were also important, with different pigeons demonstrating control by different subsets of geons; (c) recognition of the training drawings was positionally invariant; and (d) the points where the geons contacted one another were largely unimportant for picture recognition. The results provide further support for the notion that pigeons perceive both global and local aspects of complex stimuli in much the same way as do humans.
\end{abstract}

The question of whether pigeons perceive more than particulate features of complex visual stimuli still awaits a definitive answer. Cerella's (1980) early investigations were consistent with the notion that pigeons are particulate perceivers of visual stimuli and are incapable of recognizing differences in stimuli on the basis of more global, organizational properties.

Cerella trained 2 pigeons to peck in the presence of drawings of a popular Peanuts cartoon character, Charlie Brown, and to refrain from pecking in the presence of drawings of other Peanuts characters. After initial discrimination training, drawings of Charlie Brown were segmented into three portions: the head, trunk, and legs. The three parts of the figure were vertically scrambled to ensure that no two adjacent parts formed the normal body configuration. Both pigeons responded at the same high rate to the scrambled versions of Charlie Brown as they did to the intact version. Therefore, Cerella concluded that the pigeons were responding to local features alone rather than using global features, such as three-dimensional structure or overall spatial arrangement.

More recent behavioral research on avian visual perception has pointed to the dual importance of componential

Kim Kirkpatrick-Steger and Edward A. Wasserman, Department of Psychology, University of Iowa; Irving Biederman, Department of Psychology, University of Southern California.

This article is based on a doctoral dissertation submitted by Kim Kirkpatrick-Steger to the University of Iowa.

This research was supported by Grant MH47313 from the National Institute of Mental Health.

Correspondence concerning this article should be addressed to Kim Kirkpatrick-Steger, who is now at the Department of Psychology, Box 1853, Brown University, Providence, Rhode Island 02912. Electronic mail may be sent via Internet to Kim_KirkpatrickSteger@brown.edu. features and their spatial configuration for picture recognition (Brown \& Dooling, 1993; Kirkpatrick-Steger \& Wasserman, 1996; Kirkpatrick-Steger, Wasserman, \& Biederman, 1996; Steele, 1990; Wasserman, Kirkpatrick-Steger, Van Hamme, \& Biederman, 1993; Watanabe \& Ito, 1991).

A study by Wasserman et al. (1993, Experiment 1) trained pigeons with a four-choice procedure to peck a different key in the presence of each of four different line drawings: a watering can, an iron, a desk lamp, and a sailboat. Then, the pigeons saw four versions of each drawing in which the picture's components ("geons"; Biederman, 1987) were scrambled. The scrambled stimuli were created by moving the components with respect to each other but never rotating them, so that they always retained their top-bottom and left-right orientations. Accuracy to the intact drawings averaged $81 \%$ correct, whereas accuracy to the scrambled drawings averaged $52 \%$ correct. Although the decrease in accuracy to the scrambled stimuli was significant, performance did reliably exceed the chance level of $25 \%$. Thus, the results indicated a sensitivity to configural features on the part of the pigeon, but some processing of local features may have also occurred because the scrambled pictures were discriminated above chance.

An additional study replicated and extended the results of Wasserman et al. (1993). Specifically, Kirkpatrick-Steger et al. (1996) discovered that various scrambled versions of an object produced a wide range of accuracy scores, from chance $(25 \%$ correct $)$ to nearly perfect $(100 \%$ correct $)$ discrimination. That experiment used the same four drawings of the watering can, the iron, the desk lamp, and the sailboat during training. Pigeons were trained to produce different responses to each picture and then were tested with eight scrambled versions of each drawing. Cluster analyses of the accuracy scores to the individual scrambled test 
stimuli suggested that the pigeons attended primarily to the position of an individual component relative to the picture's other components. Thus, some configurations of the components were similar enough to the original drawing to allow recognition, whereas other configurations were different enough to impair picture recognition, a result that further testifies to the importance of the spatial organization of the components for picture recognition.

Although there is growing evidence that pigeons may be sensitive to the spatial organization of object features, the results of Cerella's (1980) research and those from other laboratories (Brown \& Dooling, 1993; Kirkpatrick-Steger \& Wasserman, 1996; Kirkpatrick-Steger et al., 1996; Steele, 1990; Wasserman et al., 1993; Watanabe \& Ito, 1991) need reconciling. One complication is that the critical test of sensitivity to spatial organization has involved the scrambling of stimulus parts. In scrambling a stimulus' features, there are many aspects that change: the spatial interrelations of the features, the absolute locations of the features, and the points of intersection among the features. Depending on the manner in which scrambling is done, all of these aspects may change concurrently. Thus, additional study is required to assess the contribution of various stimulus attributes to recognition of both original and scrambled images. Moreover, we have not yet directly determined the importance of the individual components to picture perception. The potential contribution of these stimulus attributes was examined here by training new pigeons to discriminate drawings of the watering can, the iron, the desk lamp, and the sailboat and then testing them with modified versions of the line drawings to examine the following issues.

First, even though the individual components appear to be necessary for picture recognition to occur, must all of the components be present? Perhaps, as with object recognition in humans, only two or three components are needed. The particular components that are present could matter as well. To assess which components participated in stimulus control, we contrasted the effect of moving or deleting an individual component with that of presenting the component alone. If an individual component were particularly important for picture recognition, then it should maintain accuracy above chance levels when presented alone, but deleting or moving it should result in measurable decrements in accuracy.

Second, in previous experiments, (Kirkpatrick-Steger et al., 1996; Wasserman et al., 1993) scrambling altered the component intersections (vertices) as well as their spatial interrelations. The components of both intact and scrambled drawings were slightly disconnected so as to remove their intersections but still maintain their qualitative relations (e.g., top-of, perpendicular-to, smaller-than). For example, the mast was separated from the hull of the sailboat, but it was still top-of, perpendicular-to, and smaller-than the hull. If the component intersections played an important role in controlling discrimination performance, then we would expect little generalization from the original to a disconnected version. Moreover, scrambling would be expected to produce a smaller effect with the weakly discriminated disconnected stimuli than with the original, strongly discriminated connected stimuli.
Third, scrambling also alters the absolute spatial position of the components. Could a change in the absolute position of the parts have accounted for the scrambling effect observed in earlier research? To assess this possibility, the intact training pictures were displayed in novel locations on the viewing screen and the four components constituting an object were displayed in separate, novel locations on the screen. The absolute position of the components across the two tests was the same, so contrasting performance to the intact versus separated stimuli should indicate the degree to which the absolute position of the components contributed to the original discrimination.

\section{Method}

\section{Animals}

Four experimentally naive feral pigeons (Columba livia) were maintained at $85 \%$ of their free-feeding weights by the delivery of 45-mg pigeon pellets (Noyes, Lancaster, $\mathrm{NH}$ ) during the experimental sessions and by supplements of mixed grain in their home cages when necessary. The pigeons were housed individually in standard pigeon cages with water and grit constantly available. The lights in the colony room were on a 14:10 light-dark cycle, with lights on at 7 a.m. and off at 9 p.m.

\section{Apparatus}

The experimental apparatus comprised four specially constructed pigeon chambers. In each chamber, one of the plywood walls contained a large opening with a metal frame attached to the outside of the box. The frame held a mylar-coated touch screen (Model 70056-001, Elmwood Sensors, Pawtucket, RI); responses on the touch screen were processed by a serial controller board (Model E271-2200, Elographics, Oak Ridge, TN). An aluminum panel in front of the touch screen permitted access to circumscribed portions of a $13-\mathrm{in}$. color video monitor located $0.9 \mathrm{~cm}$ behind the touch screen at its center. There were five openings in the panel to allow selective access to five report buttons: a $7.0-\mathrm{cm} \times 7.0-\mathrm{cm}$ square center button on which the line drawings appeared and four circular choice buttons $(1.9 \mathrm{~cm}$ in diameter) located $2.3 \mathrm{~cm}$ from each of the four corners of the central opening. The choice buttons were lit with distinctively different colors: the upper-left button was violet, the upper-right button was blue, the lower-left button was green, and the lower-right button was red. Because the monitor produces colors based on a trichromatic red-green-blue system, the colors of the choice buttons are reported as they would appear to humans. A clear Plexiglas food cup was centered on the lower rear wall of each chamber and a houselight was anchored on the upper rear wall above the food cup. A pellet magazine dispenser (Model ENV-203M, MED Associates, East Fairfield, VT), located outside of the chamber, delivered 45-mg pigeon pellets (Noyes, Lancaster, $\mathrm{NH}$ ) through a tygon tube into the food cup. The houselight and pellet dispenser were controlled by a digital input/output interface board (Model NB-DIO-24, National Instruments, Austin, TX).

Control of stimuli and recording of responses were accomplished by two Macintosh IIci and two Macintosh Quadra 650 computers. In each chamber, the pigeon's color monitor was connected to another monitor located in an adjacent room. Two of the chambers accomplished the connection with a video splitter and the other two used a distribution amplifier. Programs were developed by using HyperCard Version 2.2. 


\section{Procedure}

\section{Magazine Training}

The pigeons received from one to four sessions of magazine training in the experimental chambers. The houselight provided ambient illumination and the center button was lit yellow. The food cup was filled with pellets and the pigeon was allowed to eat all of the food; the pellets were then dispensed individually from the feeder until the pigeon reliably ate from the cup. Following magazine training, several handshaping sessions were administered, during which pecks anywhere within the five open areas of the panel were reinforced. Handshaping continued until each pigeon had pecked all five buttons on the touch screen.

\section{Pretraining}

Each pretraining session entailed 160 trials. On 80 of the trials, pecks to the lit center button resulted in reinforcement; the other 80 trials consisted of 20 presentations of each of the four circular choice buttons, with pecks to the lit button reinforced. Initially, a fixed ratio (FR) 1 pecking requirement was in place, but this requirement was gradually increased to an FR 30 . On reaching the FR 30 requirement, the pigeons were moved to discrimination training.

\section{Discrimination Training}

Pigeons were trained with a four-choice discrimination procedure. The training stimuli, pictured in Figure 1, were four line drawings depicting a watering can, an iron, a desk lamp, and a sailboat, each of which was associated with a different choice button. Each of the training stimuli contained four geons. Geons are primitive components that can be modeled by generalized cones (Marr, 1977, 1982); they are typically simple, symmetrical volumes lacking sharp concavities (e.g., cylinders, wedges, cubes). The individual geons constituting the objects were: watering can-can, handle, spout, and nozzle; iron-base, cord, handle, and knob; desk lamp—base, fixture, shade, and stem; sailboat—curved sail, hull, mast, and triangular sail.

The maximum dimension (height or width) of the four drawings was standardized to equal $2.9 \mathrm{~cm}$. Each drawing appeared in four different areas of the central viewing screen: upper-center, lowercenter, left-center, and right-center. For placement in these four locations, the drawings were centered on a line that divided the screen in half either horizontally or vertically. So, a drawing that was placed in the upper-center portion of the screen was centered in the upper half of the viewing screen on a line that vertically divided the screen in half.

The picture appeared in four different locations on the screen to encourage the pigeons to attend to all portions of the viewing screen. A pilot study from our laboratory (Kirkpatrick-Steger \& Wasserman, 1994) revealed that training with stimuli displayed in only one location resulted in poor transfer when the picture was moved to new portions of the screen, presumably because the pigeons focused on the portion containing the picture. Because some of the test stimuli appeared in novel areas of the screen, it was necessary to present the training drawings in multiple locations.

Each session comprised 160 trials given in ten 16-trial blocks. Each 16-trial block contained one presentation of each of the four objects in each of the four locations on the center button. A trial began by lighting the center button white. One peck anywhere on the center button resulted in the presentation of an object as a black line drawing on a white background. The pigeons were required to
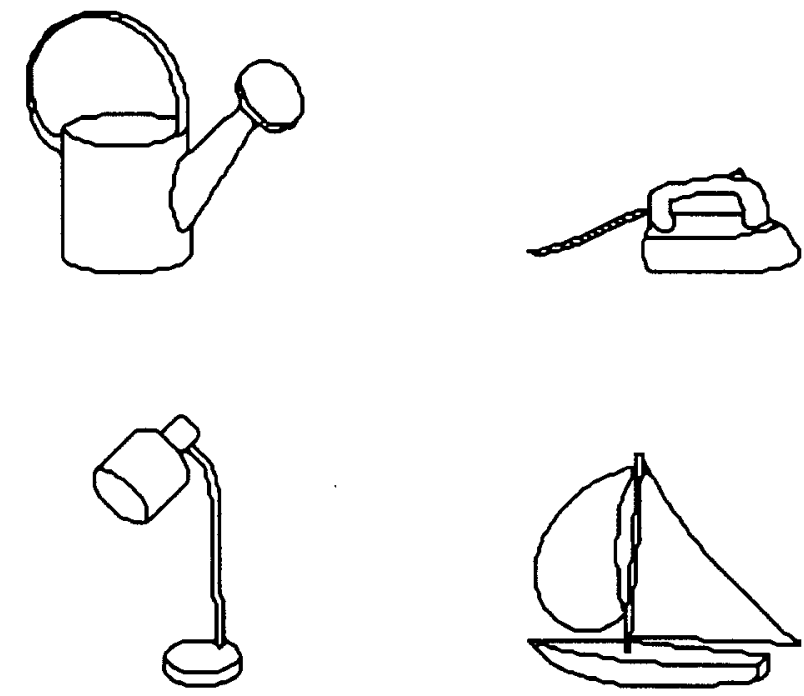

Figure 1. The watering can, the iron, the desk lamp, and the sailboat drawings used during discrimination training.

complete a set of observing responses to the pictorial stimuli, which began as an FR 5 in the early training sessions and progressively increased to an FR 30 . On completing the observing requirement, the line drawing inverted from black-on-white to white-on-black (to signal the activation of the choice buttons) and the four choice buttons were illuminated. The pigeon was then allowed to peck a single choice button. If the choice was correct, then the screen darkened and the pigeon received one or two $45-\mathrm{mg}$ pellets (to maintain the pigeon at $85 \%$ of its free-feeding weight); if the choice was incorrect, then the choice buttons darkened, the stimulus drawing reverted to black-on-white, and the trial was repeated until the correct choice occurred and food was given. 1 Only the first choice attempt of a trial was scored; correction trials were omitted from all analyses of the data. An interval varying randomly from 5 to $15 \mathrm{~s}(M=10 \mathrm{~s})$ separated trials. The pigeons continued on the discrimination task until they exceeded $70 \%$ correct to each drawing for two consecutive sessions. Then, the pigeons were individually advanced to testing.

\section{Testing}

Geon-movement versus geon-deletion test. For each of the four training drawings, four geon-movement, four 1-deletion, and four 3-deletion test stimuli were created (see Table 1 for the total number of test stimuli of each type). The three types of test drawings for the watering can are shown in Figure 2. The stimuli are arranged so that the different types of test drawings each occupy a column of the figure. Each row of the figure depicts changes to a different geon. The single geon that is moved in the left-column drawing is the one that is deleted from the middle-column drawing and is also the single remaining geon in the right-column drawing.

${ }^{1}$ Bird 3 developed an aversion to the upper-left choice button in discrimination training and was consequently required to peck the choice buttons two times following the completion of the FR 30 on both first attempts and correction trials. The two-peck choice requirement rectified the problem and was continued throughout testing. 
Table 1

Number of Individual Test Stimuli for Each Stimulus Category

\begin{tabular}{lcccc}
\hline \multicolumn{1}{c}{ Stimulus category } & Versions & Objects & Locations & Total \\
\hline Original & 1 & 4 & 4 & 16 \\
Geon-movement & 4 & 4 & 4 & 64 \\
l-Deletion & 4 & 4 & 4 & 64 \\
3-Deletion & 4 & 4 & 4 & 64 \\
Disconnected-original & 1 & 4 & 4 & 16 \\
Connected-scrambled & 4 & 4 & 4 & 64 \\
Disconnected-scrambled & 4 & 4 & 4 & 64 \\
Picture-movement & 1 & 4 & 4 & 16 \\
Geon-isolation & 24 & 4 & 1 & 96 \\
\hline
\end{tabular}

The geon-movement drawings were constructed by moving one geon $0.7 \mathrm{~cm}$ away from the other three. For the watering can, the nozzle was moved upward and rightward, the spout was moved downward and rightward, the handle was moved upward and leftward, and the can was moved downward and leftward. Similar sets of geon-movement stimuli for the iron, the desk lamp, and the sailboat were created by moving single geons $0.7 \mathrm{~cm}$ diagonally, horizontally, or vertically away from the other three geons, depending on the configuration of geons. The 1-deletion and 3-deletion drawings were constructed by erasing either one or three geons. Each of the four geons constituting each training stimulus was either erased or retained alone. The individual test stimuli appeared in the same four locations on the button as the training stimuli (upper-center, lower-center, left-center, and right-center), equating the absolute position of the intact geons.

Geon interconnection test. Four types of stimuli were presented to the pigeons: connected-original, disconnected-original, connected-scrambled, and disconnected-scrambled (see Table 1 for the total number of each type). Figure 3 displays one test stimulus for each of the four stimulus categories and for each of the training stimuli. The four original training drawings are labeled as connectedoriginal stimuli.

Four disconnected-original stimuli, one for each training stimulus, were constructed by moving the geons apart so that they no longer touched one another; all four of these stimuli are displayed in the second column of Figure 3. The disconnection of geons was accomplished by moving each geon $0.5 \mathrm{~cm}$ away from its nearest neighbor along a vector connecting their centers so that the geon interrelations remained largely intact.

Four of the eight scramblings of each training stimulus that were used in an earlier experiment (Kirkpatrick-Steger et al., 1996) were given as connected-scrambled stimuli (see Figure 4 for the complete set of connected-scrambled stimuli). These particular scramblings were chosen because they had been found previously to produce significant decrements in responding relative to the original training pictures. These 16 scramblings were also disconnected in the same manner as the disconnected-original drawings to form the set of disconnected-scrambled drawings.

The height and width of the drawings were equated for the connected-original and connected-scrambled and for the disconnected-original and disconnected-scrambled versions of each of the four training stimuli. All of the test stimuli were presented in the four locations on the button that were used in discrimination training.

Picture-movement versus geon-isolation test. The four picturemovement drawings were the watering can, the iron, the desk lamp, and the sailboat displayed in new locations on the viewing screen (see Table 1 for the total number in each stimulus category). The four new stimulus locations each involved the placement of a drawing in the center of one of the quadrants of the screen (upper-left, upper-right, lower-left, or lower-right).

The geon-isolation stimuli were created by placing the four geons in different quadrants (see the top panel of Figure 5 for a sample geon-isolation drawing of the watering can). Because there were four different geons constituting each training stimulus and four possible quadrants in which the geons could appear, the entire set of unique combinations resulted in 24 different geon-isolation drawings for each training stimulus. The geons appeared in the same position within a quadrant as in the picture-movement drawings (demonstrated in the bottom panel of Figure 5), thereby equating absolute location between the picture-movement and the geon-isolation stimuli.

General testing procedures. The three tests were administered concurrently in an attempt to distribute any cumulative effects of testing on performance across all of the test stimulus types. Each type of test (geon movement vs. geon deletion, geon interconnection, and picture movement vs. geon isolation) was given in a separate session; the test trials were intermixed with normal training trials. A session began with one block of 16 training trials. The remaining trials followed in 20-trial blocks consisting of a mixture of 16 training and 4 test trials; test stimuli occurred on Trials $5,10,15$, and 20 of a block. The geon-movement versus geon-deletion test and the geon-interconnection test each comprised 176 trials (16 training only +160 training and testing); the picture-movement versus geon-isolation test comprised 136 trials (16 training only +120 training and testing).
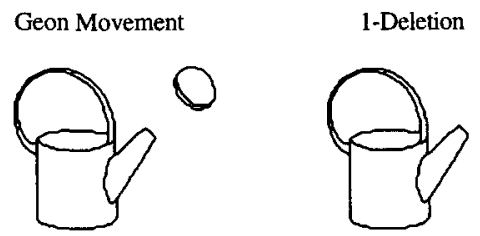

3-Deletion
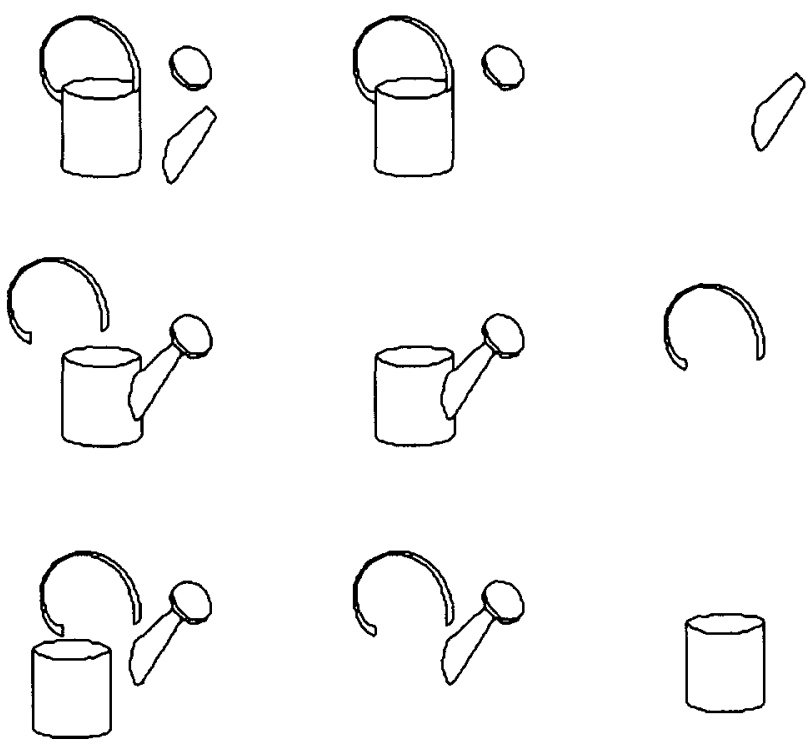

Figure 2. The geon-movement, 1-deletion, and 3-deletion drawings of the watering can used in the geon-movement versus geon-deletion test. Similar test stimuli were created for the iron, the desk lamp, and the sailboat. 


\section{Connected-}

Original
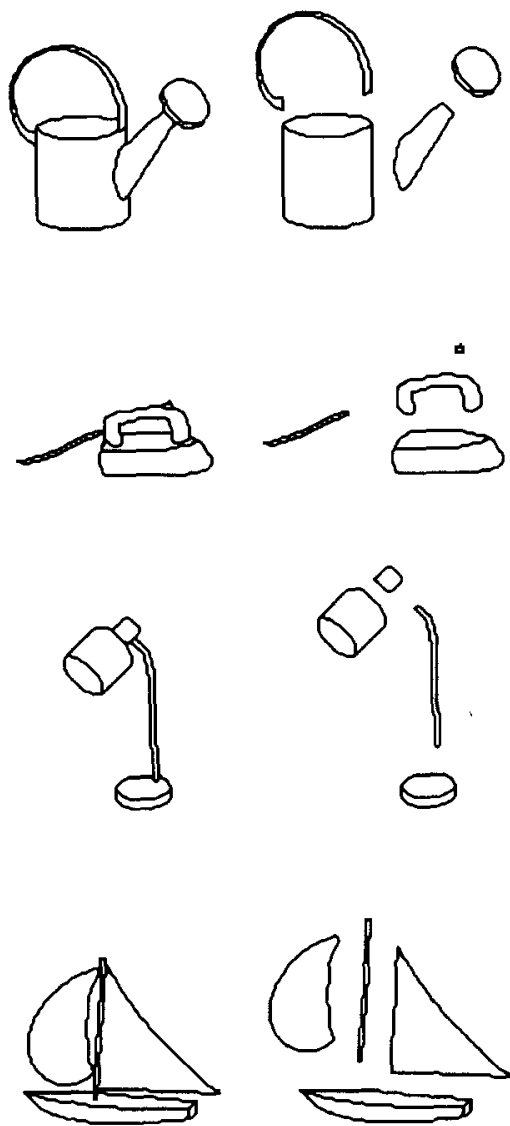

DisconnectedOriginal

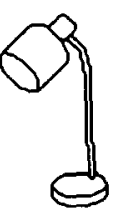

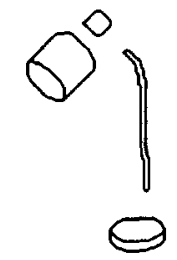

Connected-
Scrambled

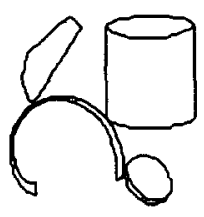

a
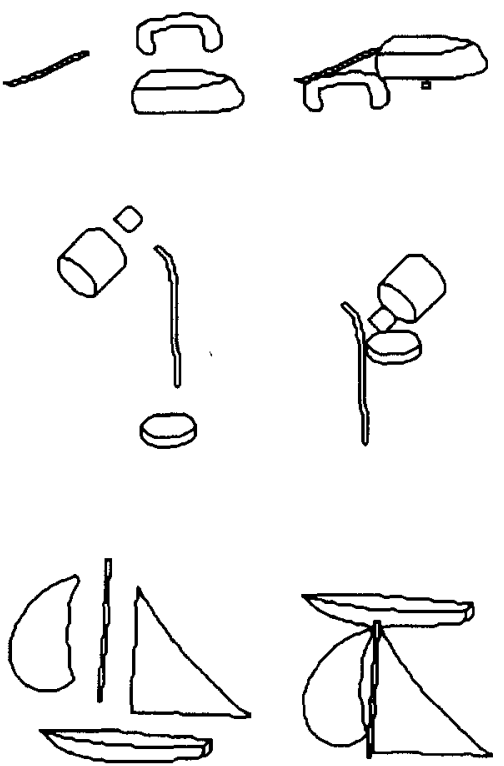

Disconnected-

Scrambled

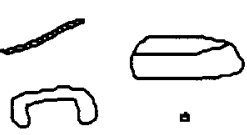

Figure 3. A subset of the connected-original, disconnected-original, connected-scrambled, and disconnected-scrambled drawings used in the geon-interconnection test.

Table 2 shows the layout of each of the three session types. Each session involved a randomly assigned subset of the test stimuli. The geon-movement versus geon-deletion test required 16 sessions; each session entailed eight original (treated as test stimuli), eight geon-movement, eight 1-deletion, and eight 3-deletion drawings. Because there were fewer original stimuli (16 total; see Table 1) than there were geon-movement (64 total), 1-deletion (64 total), and 3-deletion stimuli (64 total), the individual original stimuli were each shown four times as often as the individual members of the other three stimulus categories, thereby equating the total number of observations per stimulus category. Across the 16 geon-movement versus geon-deletion test sessions, each of the original stimuli was seen eight times in each of the four locations on the button, whereas each of the geon-movement, 1-deletion, and 3-deletion stimuli was seen twice in each location.

The geon-interconnection test also required 16 sessions; each session presented eight connected-original, eight disconnectedoriginal, eight Connected-scrambled, and eight disconnectedscrambled drawings. Across the 16 sessions, each of the connectedoriginal and disconnected-original stimuli was seen eight times in each of the four locations on the button, whereas each of the connected-scrambled and disconnected-scrambled stimuli was seen twice in each location.
The picture-movement versus geon-isolation test required 12 sessions; each session presented eight original, eight picturemovement, and eight geon-isolation drawings. Across the 12 sessions, each of the original and picture-movement stimuli was seen six times in each of its four locations on the button, whereas each of the geon-isolation test stimuli was seen only once.

As noted above, in addition to being presented on normal training trials, the original drawings were given as test stimuli as well as on training trials to equate the number of observations and conditions of reinforcement across all stimulus categories. Reinforcement followed the first choice on all test trials, regardless of whether the choice was correct. The reinforcement contingency on training trials remained the same as in discrimination training.

The entire test sequence required 48 sessions and comprised a randomly arranged series of sessions from the three tests. An individual pigeon continued through testing as long as its performance on training trials remained above $70 \%$ correct to each training picture. When accuracy levels to the training stimuli dropped below the performance criterion at any point in testing, retraining sessions were administered until accuracy again exceeded the $70 \%$ performance criterion. Each pigeon advanced through testing at its own pace. 


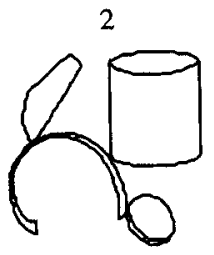

3
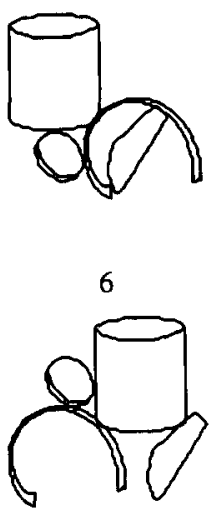

8

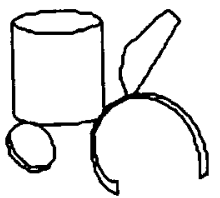

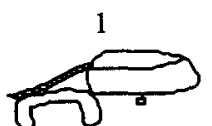
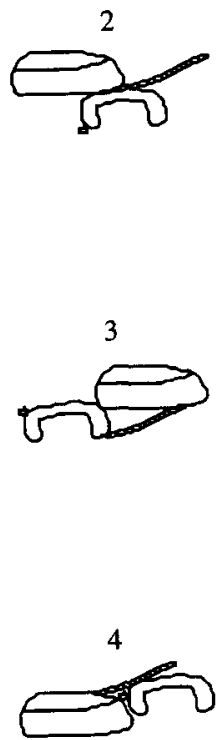

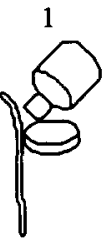

2

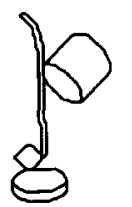

4

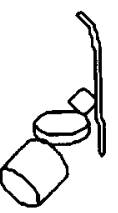

7

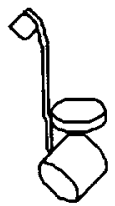

2

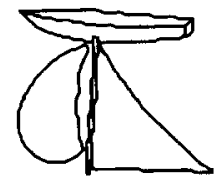

4

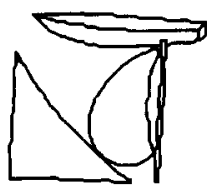

6

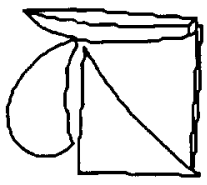

8

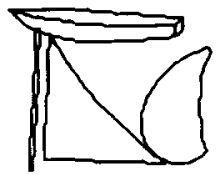

Figure 4. The full set of connected-scrambled stimuli used in the geon-interconnection test. The numbers above each scrambling are the identifications that were used by Kirkpatrick-Steger, Wasserman, and Biederman (1996).

\section{Results}

\section{Discrimination Training}

All 4 pigeons attained the $70 \%$ performance criterion to all four training pictures. On the final two sessions of training, the overall mean accuracy scores for Birds 1,2,3, and 4 were $86 \%$ (Sessions 17-18), 85\% (Sessions 31-32), $76 \%$ (Sessions 36-37), and $81 \%$ correct (Sessions $44-45$ ), respectively.

\section{Testing}

\section{Geon-Movement Versus Geon-Deletion Test}

The left portion of Figure 6 presents the mean accuracy scores to the original, geon-movement, 1-deletion, and 3-deletion test drawings; the accuracy scores were averaged across pigeons and test sessions (there were no systematic changes in performance over the course of testing). The pigeons continued to discriminate the original training stimuli when given as tests at a high level of accuracy $(86 \%$ correct). Performance on the geon-movement and 1-deletion drawings (85\% correct and $81 \%$ correct, respectively) was not discernibly less accurate than that for the originals, but there was a large drop in discrimination accuracy to the 3-deletion drawings (49\% correct). An analysis of variance (ANOVA) with variables of stimulus category (original, geon-movement, 1-deletion, and 3-deletion) and object type (watering can, iron, desk lamp, and sailboat) disclosed only a significant main effect of stimulus category, $F(3,9)=$ $72.04, p<.001$. Tukey pairwise post-hoc tests indicated that the accuracy scores to the 3-deletion drawings were significantly lower than the accuracy scores to the original, 1-deletion, and geon-movement drawings, but that the accuracy scores to the original, 1-deletion, and geonmovement drawings did not differ from one another. A binomial test revealed that the accuracy scores to all of the test stimuli, even those in the 3-deletion test, were significantly above chance $(p<.01)$.

Accuracy scores to the individual 3-deletion test stimuli are presented in Table 3 (the individual geon-movement and 1-deletion test stimuli were also examined, but accuracy scores to those stimuli were uniformly high). Scrutiny of Table 3 reveals that certain geons supported much higher accuracy levels than others. In some cases, such as the spout of the watering can, the knob of the iron, and the curved sail of the sailboat, all of the pigeons discriminated poorly when 

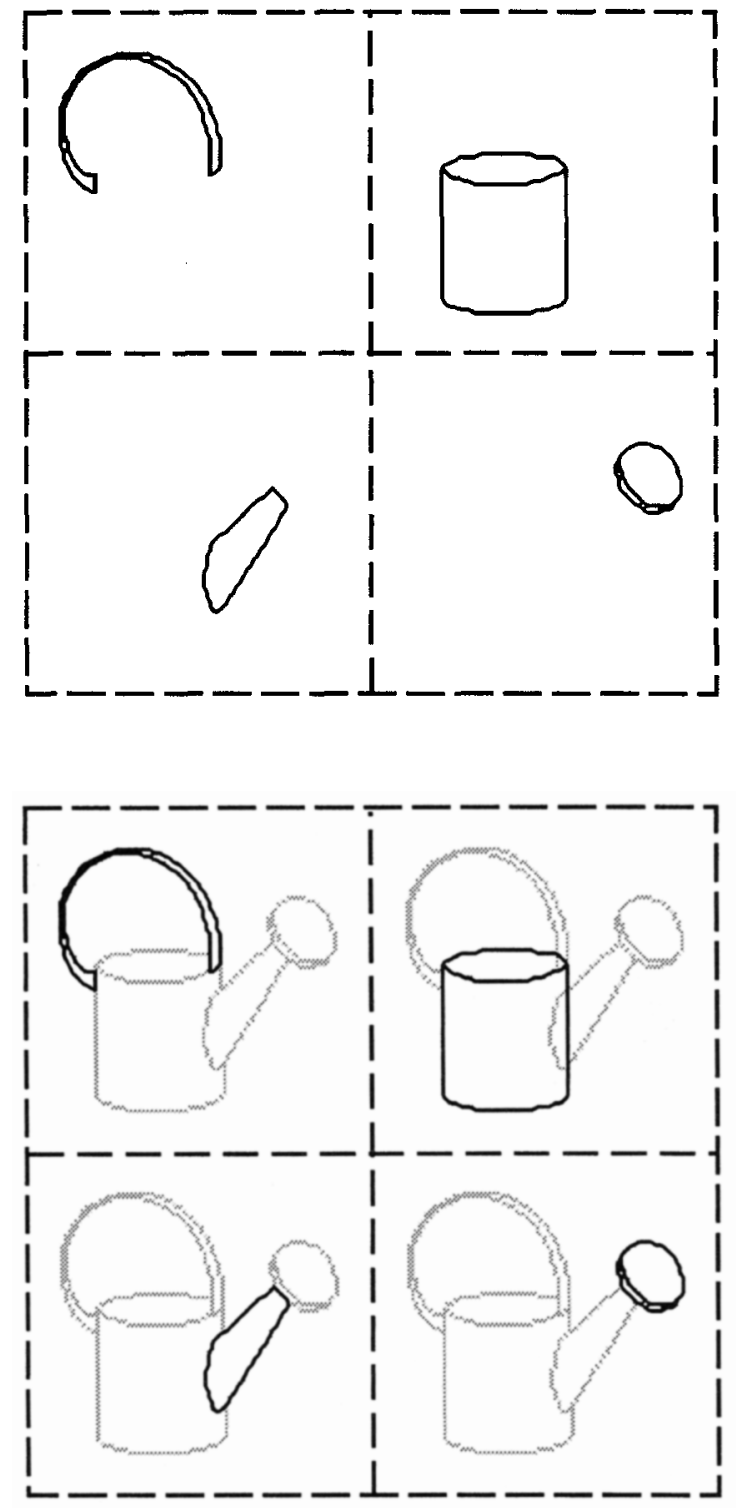

Figure 5. One of the geon-isolation drawings for the watering can. The top panel shows an arrangement of geons that the pigeons viewed. The outline of the button has been added as a dashed line for ease of presentation and was not seen by the pigeons. The bottom panel demonstrates that the geons in the top panel appeared in the same absolute location as when the entire object was moved into one of the quadrants on the picture-movement trials. The missing pieces of the watering can are presented in gray but were not seen by the pigeons.

these geons were shown by themselves. The best discriminated geons for each training object were the handle of the watering can, the cord of the iron, the base of the desk lamp, and the hull of the sailboat. However, the best discriminated geons were not the only geons discriminated above chance. In 11 out of 16 cases shown in Table 3, individual pigeons discriminated more than one geon above chance. Specifically, for the watering can, Birds 1 and 4 discriminated at
Table 2

Number of Test Stimuli in Each Stimulus Category Presented During a Session for Each of the Three Tests

\begin{tabular}{lcc}
\hline Test and stimuli & $\begin{array}{c}\text { No. per } \\
\text { session }\end{array}$ & Total \\
\hline Geon-movement vs. geon deletion (16 days) & & \\
Original & 8 & 128 \\
Geon-movement & 8 & 128 \\
1-deletion & 8 & 128 \\
3-deletion & 8 & 128 \\
Geon interconnection (16 days) & & \\
Connected-original & 8 & 128 \\
Disconnected-original & 8 & 128 \\
Connected-scrambled & 8 & 128 \\
Disconnected-scrambled & 8 & 128 \\
Picture-movement vs. geon-isolation (12 days) & & \\
Original & 8 & 96 \\
Picture-movement & 8 & 96 \\
Geon-isolation & 8 & 96 \\
\hline
\end{tabular}

least two geons above chance; for the iron, Birds $2-4$ discriminated two geons above chance; for the desk lamp, Birds 1-4 discriminated at least two geons above chance; and for the sailboat, Birds 1 and 2 discriminated at least two geons above chance.

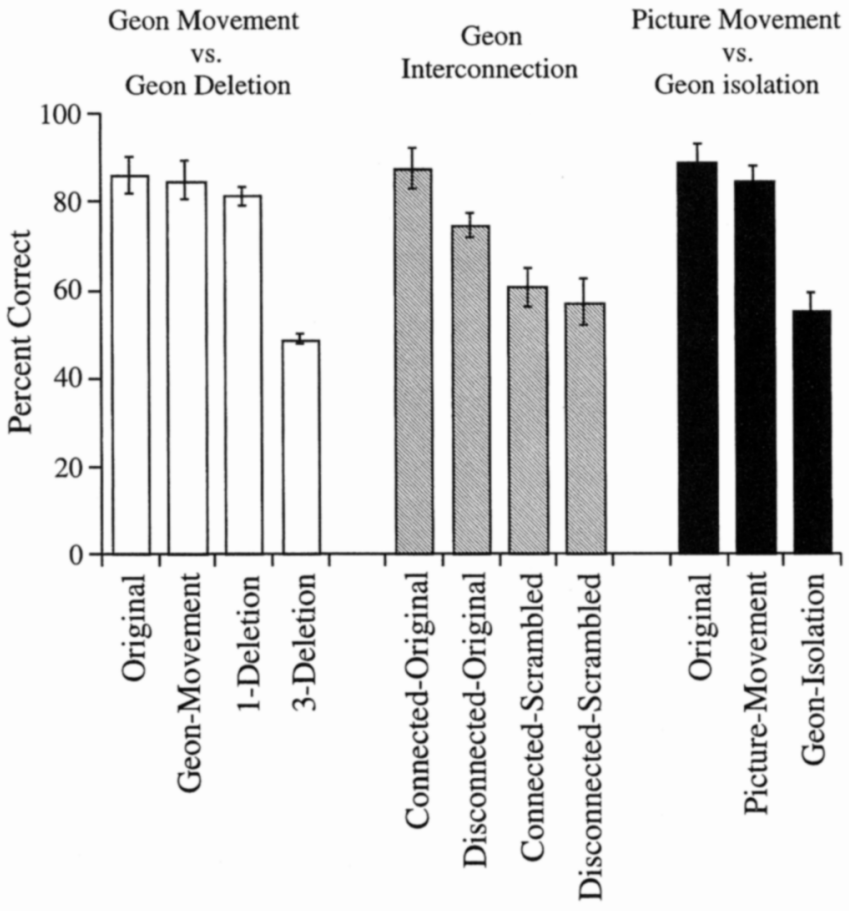

Figure 6. The overall test results from the geon-movement versus geon-deletion, geon-interconnection, and picture-movement versus geon-isolation tests. The individual bars in the figure represent the mean of the 4 pigeons plus or minus the standard error of the mean. The standard error of the mean is an index of variability between pigeons in their accuracy scores to each stimulus type. 
Table 3

Accuracy Scores Produced by Individual Pigeons to Each of the 3-Deletion Drawings, Labeled According to Which Geon Was Retained Alone on the Screen

\begin{tabular}{|c|c|c|c|c|c|}
\hline Pigeon & Original & \multicolumn{4}{|c|}{ Retained geon } \\
\hline \multicolumn{6}{|c|}{ Watering can } \\
\hline & & Can & Handle & Nozzle & Spout \\
\hline Bird 1 & $96.88 *$ & $62.50^{*}$ & $75.00 *$ & $62.50^{*}$ & 37.50 \\
\hline Bird 2 & $78.13 *$ & 0.00 & 37.50 & 37.50 & 12.50 \\
\hline Bird 3 & $93.75^{*}$ & 12.50 & $75.00^{*}$ & 12.50 & 25.00 \\
\hline Bird 4 & $78.13^{*}$ & $75.00 *$ & $75.00^{*}$ & 25.00 & 25.00 \\
\hline Mean & $86.72 *$ & 37.50 & $65.63^{*}$ & 34.38 & 25.00 \\
\hline \multicolumn{6}{|c|}{ Iron } \\
\hline & & Base & Cord & Handle & Knob \\
\hline Bird 1 & $81.25 *$ & 25.00 & 50.00 & 0.00 & 12.50 \\
\hline Bird 2 & $96.88^{*}$ & 25.00 & $75.00 *$ & $62.50^{*}$ & 12.50 \\
\hline Bird 3 & $87.50 *$ & $75.00 *$ & $62.50^{*}$ & 50.00 & 25.00 \\
\hline Bird 4 & $68.75^{*}$ & $62.50^{*}$ & $75.00 *$ & 37.50 & 25.00 \\
\hline Mean & $83.60^{*}$ & $46.88 *$ & $65.63^{*}$ & 37.50 & 18.75 \\
\hline \multicolumn{6}{|c|}{ Desk lamp } \\
\hline & & Base & Fixture & Shade & Stem \\
\hline Bird 1 & $93.75^{*}$ & $75.00 *$ & $87.50 *$ & $75.00 *$ & $62.50^{*}$ \\
\hline Bird 2 & $93.75^{*}$ & $87.50^{*}$ & 50.00 & $62.50^{*}$ & 37.50 \\
\hline Bird 3 & $90.63 *$ & $100.00^{*}$ & 50.00 & 50.00 & $87.50^{*}$ \\
\hline Bird 4 & $93.75^{*}$ & $75.00^{*}$ & 37.50 & $87.50^{*}$ & $75.00^{*}$ \\
\hline Mean & $92.97 *$ & $84.38^{*}$ & $56.25 *$ & $68.75^{*}$ & $65.63^{*}$ \\
\hline \multicolumn{6}{|c|}{ Sailboat } \\
\hline & & Curved sail & Hull & Mast & Triangle sail \\
\hline Bird 1 & $100.00^{*}$ & 0.00 & $100.00 *$ & 12.50 & $75.00^{*}$ \\
\hline Bird 2 & 93.75* & 50.00 & $100.00^{*}$ & $75.00 *$ & $87.50^{*}$ \\
\hline Bird 3 & $81.25^{*}$ & 25.00 & 37.50 & 0.00 & 50.00 \\
\hline Bird 4 & $56.25^{*}$ & 0.00 & 37.50 & 12.50 & 37.50 \\
\hline Mean & $82.81^{*}$ & 18.75 & $68.75^{*}$ & 25.00 & $62.50^{*}$ \\
\hline
\end{tabular}

Note. Accuracy scores to the original drawings are included for reference.

*Significantly above $25 \%, p<.05$.

Although some geons were discriminated above chance when they were presented alone, even the best geons failed to sustain accuracy at the level of the original drawing. It can be seen in Table 3 that, in 12 out of 16 instances, the individual pigeons' accuracy scores to the whole drawing exceeded accuracy scores to the single geon controlling the highest level of discrimination. This difference, in combination with the fact that deleting one geon had no significant effects on accuracy, suggests that more of the picture than a single salient part was responsible for the pigeons' recognition of the intact drawings, a conclusion that is consistent with the fact that more than one component often controlled reliable discrimination performance.

\section{Geon Interconnection Test}

The overall results of the geon-interconnection test are shown in the middle portion of Figure 6. The disconnection of the geons constituting the original objects did produce a modest decrement in accuracy scores $(75 \%$ correct) relative to the connected-original drawings ( $88 \%$ correct), but the decrement was much smaller than that to the connectedscrambled stimuli ( $61 \%$ correct). The disconnected-scrambled drawings were discriminated nearly as well as the connectedscrambled drawings (57\% correct). All of the drawings were discriminated significantly above chance $(p<.01)$.

An ANOVA with variables of object type, connection versus disconnection, and original versus scrambled disclosed a significant main effect of original versus scrambled, $F(1,3)=22.2, p<.05$, but did not reveal a significant main effect of object type, a main effect of connection versus disconnection, or any interaction of object type, connection versus disconnection, or original versus scrambled with one another. Therefore, as a main effect, geon disconnection (and any consequent disruption of geon intersections) did not produce any major effects on picture recognition. Moreover, the lack of a significant interaction between connection 
versus disconnection and original versus scrambled indicates that the $13 \%$ decrement in accuracy to the disconnectedoriginal drawings was not reliable. On the other hand, geon scrambling (and any consequent disruption of geon interrelations) did produce significant decrements in discrimination accuracy. These two results suggest that the geon intersections were not salient features for picture recognition. The spatial organization of the components was, however, critical for accurate picture recognition. Moreover, the presence of the geons themselves may have played a role because the scrambled stimuli were recognized above chance $(p<.01)$.

\section{Picture-Movement Versus Geon-Isolation Test}

The overall accuracy scores to the original, picturemovement, and geon-isolation test stimuli are presented in the right portion of Figure 6. Moving the entire picture had no discernible effect on discrimination accuracy $(85 \%$ correct) relative to the original training pictures $(89 \%$ correct), but there was a significant generalization decrement to geon isolation drawings $(56 \%$ correct). However, accuracy scores to all of the test stimuli were above chance $(p<.01)$. An ANOVA with variables of stimulus category and object type disclosed only a significant effect of stimulus category, $F(2,6)=26.50, p<.001$. Pairwise Tukey post-hoc tests revealed that the geon-isolation drawings produced significantly lower accuracy scores than did the original and picture-movement drawings $(p<.05)$; the original and picture-movement drawings did not differ from one another.

\section{Comparison of the Three Tests}

The present experiment was designed to pinpoint the principal controlling elements of the line drawings that contributed to their recognition by pigeons. To further address this concern, the mean accuracy scores to each type of test drawing were ranked in decreasing order. The scores are presented in Table 4, along with a description of the major alterations that were made to each drawing. (The list of drawing characteristics was not intended to be exhaustive.) The drawings were categorized as follows: (a) the number of geons present, varying from 1 to 4 ; (b) the number of original geon interrelations preserved, varying from 0 to 4 ; (c) the number of original geon intersections preserved, varying from 0 to 4 ; (d) the number of geons appearing in their original absolute locations, varying from 0 to 4; and (e) the degree of compactness when four geons were present (the most compact versions were assigned a score of 4 , and progressively less compact drawings were assigned scores from 3 to 1 ).

A one-way ANOVA with a variable of stimulus category was conducted on the mean accuracy scores produced by each pigeon to each of the stimulus categories; it yielded a significant main effect, $F(8,24)=20.77, p<.001$. A Newman-Keuls post hoc test revealed that accuracy scores to the original, geon-movement, picture-movement, and 1-deletion drawings were significantly greater than accuracy scores to the connected-scrambled, disconnected-scrambled, geon-isolation, and 3-deletion drawings. Accuracy scores to the disconnected-original drawings did not differ reliably from any of the higher accuracy scores, but they were significantly greater than accuracy scores to the disconnectedscrambled, geon-isolation, and 3-deletion drawings.

Thus, examination of the relationship between accuracy scores and stimulus characteristics revealed the following: (a) When only one geon was present, accuracy was the worst; however, accuracy was only $7 \%$ lower than when all four geons were present in the geon-isolation drawings. Thus, the number of geons did not by itself account for the overall pattern of accuracy scores. (b) When no geon relations were preserved (connected-scrambled, disconnected-scrambled, geon-isolation, and 3-deletion drawings), accuracy scores ranged from $49 \%$ correct to $61 \%$ correct. When three or more relations were intact, accuracy ranged from $75 \%$ correct to $88 \%$ correct. Thus, the number of relations that were preserved appeared to be a strong contributor to performance. (c) Disconnecting the geons in the original drawings did not produce a large effect on accuracy. Thus, the number of intersections preserved may have contributed slightly to picture recognition, but it was not a major determinant. (d) Preserving the absolute location of the geons was not necessary. The original drawings where the absolute location of all four geons was preserved provided no significant advantage in discrimination accuracy over the picture-movement drawings where the absolute location of all four geons changed. (e) The compactness of the geons was not strongly related to accuracy. In particular, the connected-scrambled drawings, which received a compactness score of 4 , produced accuracy scores that were similar to the disconnected-scrambled and geonisolation drawings, which received compactness scores of only 2 and 1 , respectively.

\section{Discussion}

The movement or deletion of a single geon produced no discernible effects on accuracy scores, whereas the deletion of three geons produced significant decrements in picture recognition accuracy. Thus, at a minimum, the pigeons must have attended to two geons or else there would have been systematic effects of moving or deleting one geon. Although deleting three geons had a large effect on accuracy, some individual geons supported above-chance picture recognition when they were presented alone. In the majority of cases, more than one geon sustained accuracy levels above chance. However, the set of individual geons that yielded above-chance accuracy scores was largely idiosyncratic for the individual pigeons.

Disrupting the spatial interrelations by scrambling the geons had significant detrimental effects on accuracy, but disrupting the geon intersections by small detachments did not significantly affect discrimination accuracy. In the original drawings, there are several features that are formed when two or more geons contact one another. Because each training drawing contained a unique set of intersections, such features could have provided a means for recognizing the drawings. In the disconnected-original stimuli, the 
Table 4

Mean Accuracy Scores for Each of the Stimulus Categories

\begin{tabular}{|c|c|c|c|c|c|c|}
\hline Drawing & $\begin{array}{c}\text { Mean } \\
\text { accuracy }\end{array}$ & $\begin{array}{l}\text { Geons } \\
\text { present }\end{array}$ & $\begin{array}{l}\text { Relations } \\
\text { preserved }\end{array}$ & $\begin{array}{l}\text { Intersections } \\
\text { preserved }\end{array}$ & $\begin{array}{l}\text { Location } \\
\text { preserved }\end{array}$ & $\begin{array}{c}\text { Compactness } \\
\text { of } 4 \text { geons }{ }^{\mathrm{a}}\end{array}$ \\
\hline Original $^{b}$ & 87.63 & 4 & 4 & 4 & 4 & 4 \\
\hline Geon-movement & 84.97 & 4 & 3 & 3 & 3 & 3 \\
\hline Picture-movement & 84.90 & 4 & 4 & 4 & 0 & 4 \\
\hline 1-Deletion & 81.44 & 3 & 3 & 3 & 3 & \\
\hline Disconnected-original & 74.61 & 4 & 4 & 0 & 0 & 2 \\
\hline Connected-scrambled & 60.55 & 4 & 0 & $0^{c}$ & $\mathbf{0}$ & 4 \\
\hline Disconnected-scrambled & 57.23 & 4 & 0 & 0 & 0 & 2 \\
\hline Geon-isolation & 55.99 & 4 & 0 & 0 & 0 & 1 \\
\hline 3-Deletion & 48.83 & 1 & 0 & 0 & 1 & \\
\hline
\end{tabular}

The most compact drawings were assigned a score of 4 , and progressively less compact drawings were assigned scores from 3 to 1. b Averaged across the three tests. ${ }^{\mathrm{c}}$ Assumed to be 0 , although on rare occasion an intersection in a scrambling may have resembled an intersection in the original drawing.

original relations of the components were similar to the connected-original stimuli, but the geon intersections were disrupted. Because the identification of the original drawings was not significantly disrupted by the operation of disconnection, it can be concluded that geon intersections did not exert substantial control over discriminative behavior (although it is possible that the pigeons used projected vertices in the disconnected-original stimuli; see Waltz, 1975). These results accord with an earlier finding that pigeons do not strongly attend to the identity of line segments or vertices (Van Hamme, Wasserman, \& Biederman, 1992).

Moving the entire picture to a new location had no effect on recognition accuracy, but separately moving the geons to new locations produced a significant impairment. Because the picture-movement drawings were recognized as well as the original training stimuli, the absolute location of the geons on the viewing screen must have had no effect on picture recognition, that is, picture recognition by the pigeons was positionally invariant. That the absolute position of components on the screen would play a part in picture recognition is unlikely because the pigeon was free to move about as it observed the images. Moreover, the drawings appeared in four separate locations over the course of training and testing, which should have discouraged the pigeons from learning the placement of the geons relative to the screen boundaries.

Finally, the attempt to relate accuracy scores to stimulus characteristics (see Table 4) highlighted the importance of geon interrelations. When the geon interrelations were disrupted, the largest decrements in accuracy scores occurred, but accuracy still remained above chance. Thus, the presence of one or more geons or their constituent features (e.g., line contours) must have supported the above-chance accuracy levels. Other factors, such as geon intersections, the absolute location of the geons, and the compactness of the geons did not appear to have contributed substantially to picture recognition. The present series of tests points to the dual importance of features and their organization to picture recognition, a result that is consistent with earlier studies on the subject (Brown \& Dooling, 1993; Kirkpatrick-Steger \&
Wasserman, 1996; Kirkpatrick-Steger et al., 1996; Steele, 1990; Wasserman et al., 1993; Watanabe \& Ito, 1991).

\section{Relation to Previous Studies of Pigeon Picture Perception}

The above findings lead to the interpretation that pigeons do attend to line drawings in both a local and global sense. Taken in the context of two other lines of investigation using similar stimuli and training methods (Van Hamme et al., 1992; Wasserman et al., 1996), these results suggest that pigeons may perceive stimuli in much the same manner as humans do.

Wasserman et al. (1996) tested whether pigeons could recognize line drawings of objects despite variations in viewpoint. The four-choice method was used to train a discrimination among line drawings of an airplane, a chair, a desk lamp, and a flashlight. Three groups of pigeons were exposed to the four objects in either $0^{\circ}, 33^{\circ}$, or $67^{\circ}$ orientations. Then, the pigeons were tested with novel versions of the four objects that were rotated in the depth plane from $-100^{\circ}$ to $167^{\circ}$ in $33^{\circ}$ increments. All three groups produced similar results: (a) The reinforced training orientation was discriminated at the highest level of accuracy; (b) a decremental generalization gradient was observed for the test orientations, with a gradual decline in accuracy as a function of distance from the trained orientation; and (c) all test orientations were recognized above chance. Thus, after training with only one orientation, pigeons were able to recognize novel orientations despite rotations of up to $133^{\circ}$ on either side of the training value. Such viewpoint invariant recognition is quite remarkable because rotation in the depth plane resulted in a change in the parts of the object that were seen by the pigeons. As objects were rotated farther from the training vantage point, the original parts became occluded as new parts came into view. To have recognized an object over such a wide range of orientations, the pigeons must have attended to more than the particulate aspects of the line drawings.

Van Hamme et al. (1992) conducted three experiments to examine whether pigeons recognized pictures on the basis of 
particulate features such as pieces of contour or vertices. Pigeons were trained with a four-choice task to discriminate line drawings of four objects in which $50 \%$ of the contours and vertices were deleted. In humans, these stimuli would support object recognition, presumably because enough information remains to extract the individual components and their interrelations. Then, pigeons were tested with complementary contours that were presented in their spatially appropriate positions on the screen or with the original or complementary contours in spatially scrambled arrangements. The complementary images were discriminated near the level of the original images, albeit with some generalization decrement, whereas the scrambled contours and vertices were recognized much more poorly. Still, the scrambled complementary contours were capable of supporting abovechance recognition for some objects, possibly because of generalization from the original contours to the similar complementary contours. In sum, the results indicated that the pigeons were sensitive to both local and global aspects of the line drawings.

\section{Theories of Picture Perception}

Several theories may account for the above results. The most discriminating aspect of the theories is how representations of objects are stored. Three major types of theories that have been previously applied to pigeon visual perception are considered below: particulate-feature, template, and primitive models.

Although there are many different types of feature models, a prominent version in the area of pigeon visual perception is Cerella's particulate-feature theory. Drawing on a series of investigations that attempted to specify the properties of pictorial stimuli that control discriminative behavior in pigeons (Cerella, 1977, 1979, 1980, 1982, 1986, 1990), Cerella concluded that the pigeon is a particulate perceiver of visual information. Specifically, he proposed that pigeons represent objects as random, unorganized collections of local features. Object recognition will occur provided that enough of the features are present, regardless of their spatial position or interrelation to one another.

Template models (e.g., Edelman \& Bülthoff, 1992; Poggio \& Edelman, 1990; Rock, 1973; Tarr, 1995) argue that multiple representations of an object may exist, each varying from the others with respect to viewpoint, size, position, and so on. An object can be recognized either by an exact match with a stored template or by generalization to similar templates completed through the process of smooth deformations of the template, with a modest cost in time and accuracy.

Finally, primitive theories of object recognition purport that images are stored in the form of structural descriptions which contain a specification of the object's primitive components and their organization. There are several primitive theories, which differ primarily in the type of primitive that is used by the visual system. Recognition-by-components (RBC; Biederman, 1987; Hummel \& Biederman, 1992) is a primitive theory in which geons serve as the elementary components. The major contribution of RBC is that it proposes a precise and reasonably limited set of components derived directly from known perceptual mechanisms such as good continuation, symmetry, and Pragnanz. Object recognition occurs as follows: Edges are extracted and nonaccidental properties are detected (which in turn facilitates the further detection of edges), parsing of an object into its constituent geons occurs at regions of concavity, geons are identified, and the geons and their spatial interrelations are matched against stored structural descriptions of objects (which include a specification of both the identity and interrelation of constituent geons) until a sufficiently good match is found.

\section{Discriminating Among Theoretical Accounts}

Drawing from the present results, as well as from previous work using similar line drawings (Kirkpatrick-Steger et al., 1996; Van Hamme et al., 1992; Wasserman et al., 1993, 1996), there are six major facts of pigeon picture perception that must be accounted for theoretically.

1. Picture recognition is largely viewpoint invariant (Wasserman et al., 1996).

2. Picture recognition requires more than just individual pieces of contour or vertices-these features must appear in the appropriate spatial concatenation (Van Hamme et al., 1992).

3. Picture recognition is positionally invariant (picturemovement versus geon-isolation test).

4. The geons must appear in their original spatial concatenation for highly accurate picture recognition to occur (Kirkpatrick-Steger et al., 1996; Wasserman, et al., 1993; geon-interconnection test).

5. More than one geon is necessary for highly accurate picture recognition, but all four geons do not have to be present (geon-deletion versus geon-movement test).

6. Particulate features such as the geons, their constituent pieces of contour, or their vertices can support abovechance recognition, but with a considerable generalization decrement relative to spatially intact versions (KirkpatrickSteger et al., 1996; Van Hamme et al., 1992; Wasserman et al., 1993; geon-interconnection test).

Cerella's (1986) particulate-feature theory has the most trouble in accounting for the above facts. There are several definite predictions from particulate-feature theory: (a) Picture recognition can be viewpoint invariant, but only if the same features are present in all viewpoints; (b) picture recognition should be unaffected by scrambling object features; and (c) pigeons should not be able to recognize a complementary image because it would not activate the previously learned parts. In his investigations with pigeons, Cerella has reported results consistent with the above conclusions. For example, pigeons failed to generalize responding to stimuli rotated in depth (Cerella, 1977), but showed no decrement in recognizing images that were spatially scrambled (Cerella, 1980). As such, many of the results from our laboratory are at odds with Cerella's earlier research and theoretical renderings (see Wasserman et al., 1993, 1996, for discussions of possible reasons for the differences in experimental outcomes).

The most damaging criticism of particulate-feature theory 
is that there is no allowance for the pigeon's ability to discriminate stimuli that differ only in the spatial organization of their features. Humans can readily discriminate objects that contain the same components in different arrangements, such as a cup and a pail; both contain a cylinder and a handle, but the handle attaches on top of the pail and it attaches to the side of the cup. In pigeons, there are two pieces of evidence demonstrating a successful discrimination between different spatial formations of components (Kirkpatrick-Steger \& Wasserman, 1996; Wasserman et al., 1993, Experiment 2). Particulate-feature theory cannot account for these results nor can it account for the substantial generalization decrement to scrambled stimuli.

Template theories fare better in accommodating the above empirical facts. For example, object recognition should be somewhat viewpoint invariant but not completely so. Training pigeons to recognize a single orientation of an object should result in a generalization gradient such as that obtained by Wasserman et al. (1996). The stored template of one viewpoint should allow generalization to nearby orientations but not to highly different orientations. Template models also predict that scrambling object parts should result in poor recognition because the scrambled versions would represent an inadequate match to the stored templates. Similarly, deleting three geons should have a larger decremental effect than deleting one geon because a single geon would represent a poorer match to the stored template.

Although template models appear to account for most of the pigeon perception data, they have problems in dealing with shape priming phenomena in human visual perception research (see Hummel \& Biederman, 1992, and Pinker, 1984 , for further discussion of template models). There are two major criticisms of template models: (a) When large numbers of objects need to be stored and discriminated, the critical alignment features used to select candidate templates may be difficult to obtain; and (b) a template model must have a stored representation of an object before it can classify two views of it as equivalent, that is, template models cannot account for viewpoint invariance in unfamiliar objects. Whether these same criticisms are relevant to pigeon visual perception remains to be seen.

RBC also easily handles the six facts discussed at the beginning of this section. For example, object recognition should be invariant with respect to viewpoint and position, provided that a sufficient subset of the geons and their spatial interrelations can be recovered. RBC is not at odds with the observed generalization decrement in the three-dimensional rotation studies because the rotated versions sometimes resulted in the display of new parts; thus, the extreme rotations would have a different structural description from the original orientations. As Wasserman et al. (1996, Experiment 2a) found, training with three orientations that were highly discrepant with respect to viewpoint $\left(-100^{\circ}, 33^{\circ}\right.$, and $167^{\circ}$ ) resulted in a generalization gradient that was relatively flat over the entire range of orientations. Thus, training the pigeons with viewpoints that displayed all of the geons allowed for much better recognition over the entire rotational range. Additional experimental results can be readily explained by RBC. For example, disrupting the spatial interrelations of the geons should have a large effect on accuracy because the stored structural description of an object contains a specification of both its geons and their relational aspects (e.g., top-of, larger-than). Similarly, deleting three geons should have a much greater effect on recognition accuracy than deleting one geon. These results can be accounted for by a Weber activation function, in which the net input $=$ number of matching parts $/(k+$ the number of expected parts), where $k$ is the Weber constant (Hummel \& Biederman, 1992). Here, the number of expected parts is four because each of the training objects contained four geons. Although the Weber constant is unknown for pigeons, the function would capture the large jump in accuracy when three parts were present as compared to when only one part was present. Finally, there should be reasonably good generalization from a stimulus containing half the contours and vertices to its complementary image, provided that enough of the vertices and contours were present to allow for activation of the whole object's geons.

Whether template or primitive models will prove better in accommodating many different pigeon visual perception phenomena remains to be seen. Further investigations with stimuli that test additional object properties will undoubtedly allow for further discrimination between the theories. One interesting possible test would be to examine the pigeon's sensitivity to geon versus metric variations (see Biederman \& Bar, 1995, for results with human participants). Moreover, differences in the degree to which geons may be extracted from images (e.g., outlines, silhouettes) could provide an additional basis for selecting one model over the other.

Regardless of whether template or primitive theories prove better in accommodating the results, it appears that many of the properties of visual perception in the pigeon are similar to those found in humans (see Biederman \& Gerhardstein, 1995, for a review of human object-recognition studies, and Wasserman, 1991, 1993, for comparable research with pigeons). The present research, along with previous investigations of avian visual perception, points to the interesting possibility that a unified theory of visual perception that does not require special provisions for different species of animals may be achievable.

\section{References}

Biederman, I. (1987). Recognition-by-components: A theory of human image understanding. Psychological Review, 94, $115-147$.

Biederman, I., \& Bar, M. (1995, November). One-shot viewpoint invariance with nonsense objects. Paper presented at the Annual Meeting of the Psychonomic Society, Los Angeles, CA.

Biederman, I., \& Gerhardstein, P. C. (1995). Viewpoint-dependent mechanisms in visual object recognition: Reply to Tarr and Bülthoff (1995). Journal of Experimental Psychology: Human Perception and Performance, 21, 1506-1514.

Brown, S. D., \& Dooling, R. J. (1993). Perception of conspecific faces by budgerigars (Melopsittacus Undulatus): II. Synthetic models. Journal of Comparative Psychology, 107, 48-60.

Cerella, J. (1977). Absence of perspective processing in the pigeon. Pattern Recognition, 9, 65-68. 
Cerella, J. (1979). Visual classes and natural categories in the pigeon. Journal of Experimental Psychology: Human Perception and Performance, 5, 68-77.

Cerella, J. (1980). The pigeon's analysis of pictures. Pattern Recognition, 12, 1-6.

Cerella, J. (1982). Mechanisms of concept formation in the pigeon. In D. J. Ingle, M. A. Goodale, \& R. W. Mansfield (Eds.), Analysis of visual behavior (pp. 241-263). Cambridge, MA: MIT Press.

Cerella, J. (1986). Pigeons and perceptrons. Pattern Recognition, 19, 431-438.

Cerella, J. (1990). Shape constancy in the pigeon. In M. L. Commons, R. J. Herrnstein, S. M. Kosslyn, \& D. B. Mumford (Eds.), Quantitative analyses of behavior: Behavioral approaches to pattern recognition and concept formation (Vol. 8, pp. 145-163). Hillsdale, NJ: Erlbaum.

Edelman, S., \& Bülthoff, H. H. (1992). Orientation dependence in the recognition of familiar and novel views of 3D objects. Vision Research, 32, 2385-2400.

Hummel, J. E., \& Biederman, I. (1992). Dynamic binding in a neural network for shape recognition. Psychological Review, 99 , 480-517.

Kirkpatrick-Steger, K., \& Wasserman, E. A. (1994). [Positional invariance in the pigeon]. Unpublished raw data.

Kirkpatrick-Steger, K., \& Wasserman, E. A. (1996). The what and the where of the pigeon's processing of complex visual stimuli. Journal of Experimental Psychology: Animal Behavior Processes, 22, 60-67.

Kirkpatrick-Steger, K., Wasserman, E. A., \& Biederman, I. (1996). Effects of spatial rearrangement of object components on picture recognition in pigeons. Journal of the Experimental Analysis of Behavior, 65, 465-475.

Marr, D. (1977). Analysis of occluding contour. Proceedings of the Royal Society of London, 197B, 441-475.

Marr, D. (1982). Vision. San Francisco, CA: Freeman.

Pinker, S. (1984). Visual cognition: An introduction. Cognition, 18, $1-63$.

Poggio, T., \& Edelman, S. (1990). A network that learns to recognize 3D objects. Nature, 343, 263-266.

Rock, I. (1973). Orientation and form. San Diego, CA: Academic Press.
Steele, K. M. (1990). Configural processes in pigeon perception. In M. L. Commons, R. J. Herrnstein, S. M. Kosslyn, \& D. B. Mumford (Eds.), Quantitative analysis of behavior: Behavioral approaches to pattern recognition and concept formation (Vol. 8, pp. 111-125). Hillsdale, NJ: Erlbaum.

Tarr, M. J. (1995). Rotating objects to recognize them: A case study on the role of viewpoint dependency in the recognition of three-dimensional objects. Psychonomic Bulletin and Review, 2, 55-82.

Van Hamme, L. J., Wasserman, E. A., \& Biederman, I. (1992). Discrimination of contour-deleted images by pigeons. Journal of Experimental Psychology: Animal Behavior Processes, 18, 387399.

Waltz, D. (1975). Understanding line drawings of scenes with shadows. In P. H. Winston (Ed.), The psychology of computer vision (pp. 19-91). New York: McGraw Hill.

Wasserman, E. A. (1991). The pecking pigeon: A model of complex visual processing. [Review of Quantitative analyses of behavior, Vol. 8: Behavioral approaches to pattern recognition and concept formation]. Contemporary Psychology, 36, 605-606.

Wasserman, E. A. (1993). Picture perception: A bird's eye view. Current Directions in Psychological Science, 2, 184-189.

Wasserman, E. A., Gagliardi, J. L., Cook, B. R., Kirkpatrick-Steger, K., Astley, S. L., \& Biederman, I. (1996). The pigeon's recognition of drawings of depth-rotated stimuli. Journal of Experimental Psychology: Animal Behavior Processes, 22, 205-221.

Wasserman, E. A., Kirkpatrick-Steger, K., Van Hamme, L. J., \& Biederman, I. (1993). Pigeons are sensitive to the spatial organization of complex visual stimuli. Psychological Science, 4, 336-341.

Watanabe, S., \& Ito, Y. (1991). Discrimination of individuals in pigeons. Bird Behaviour, 9, 20-29.

Received August 26, 1996

Revision received March 3, 1997

Accepted March 4, 1997 